\title{
COnE).(OES
}

CIÊNCIA E TECNOLOGIA

\section{INTERNACIONALIZACIÓN DE LAS INSTITUCIONES DE NIVEL SUPERIOR. FLUJOS DE MOVILIDAD ESTUDIANTIL.}

\author{
MARCOS JAVIER ANDRADA \\ UNLAR - Universidad Nacional de La Rioja \\ $<$ licmarcosandrada@yahoo.com.ar> \\ DOI: <https://doi.org/10.21439/conexoes.v14i5.1765>
}

\begin{abstract}
Resumo. Este artículo se trata de un estudio de tipo descriptivo cuyo objetivo es el análisis del flujo de movilidad de los alumnos de 5 países de la región de Latinoamérica. El objetivo del mismo es comprender la dimensión y el sentido de los flujos migratorios de los estudiantes de los países de la región. Se utilizará una metodología comparativa con fuentes de datos secundarias con indicadores provistos por la oficina de estadísticas de la UNESCO. Se espera con este análisis contribuir al conocimiento del volumen y el sentido de los flujos migratorios de estudiantes y las posibles implicancias que la movilidad provoca tanto en los lugares de orígenes como en los de destino.
\end{abstract}

Palavras-chaves: Flujo estudiantil. Movilidad. Universidad. Internacionalización. Estudios superiores.

\section{INTERNATIONALIZATION OF HIGHER LEVEL INSTITUTIONS. STUDENT MOBILITY FLOWS}

\begin{abstract}
This article is a descriptive study whose objective is the analysis of the mobility flow of students from 5 countries in the Latin American region. Its objective is to understand the dimension and meaning of the migratory flows of students from the countries of the region. A comparative methodology with secondary data sources will be used with indicators provided by the UNESCO statistics office. It is expected with this analysis to contribute to the knowledge of the volume and the meaning of the migratory flows of students and the possible implications that mobility causes both in the places of origins and in those of destination.
\end{abstract}

Keywords: University. Student flow. Mobility. Internationalization. Higher education.

\section{INTRODUÇÃO}

Sylvie Didou Aupetit señala que: "por internacionalización de la educación superior debe entenderse el esfuerzo sostenido y sistemático para hacer que la educación superior responda ante los requisitos y desafíos relacionados con la globalización de las sociedades, economía, trabajo y mercados, para aportar, de esta manera, una visión instrumental de la internacionalización de la Educación Superior" (AUPETIT, 2007).

La internacionalización de la Educación superior es un fenómeno complejo que comprende diversos aspectos que tales como la movilidad académica, el ofrecimiento de propuestas de programas académicos fuera de la jurisdicción política e incluso la creación de institucio- nes o asociaciones transnacionales que forman parte de este mundo globalizado. La movilidad estudiantil es, quizás, la faceta más visible de la internacionalización, que muestra su situación más radical en la fuga de cerebros que implica la radicación de individuos altamente formados en países que invirtieron escasamente en su formación pero que requieren de ese nivel de calificación para su desarrollo y pueden ofrecer mejores condiciones laborales y personales que las que los individuos tienen en su lugar de origen.

Los dos antecedentes más importantes, ineludibles, para hablar de la internacionalización de la educación superior son el acuerdo de Bolonia, surgida en el seno de la comunidad europea en tanto comunidad política 
de derecho que comenzó su génesis como un tratado comercial al que luego se le fueron sumando otros acuerdos como este referido a la educación de nivel superior, por un lado. Otro antecedente importante reconocido también por Córtez Chávez es la declaración de la Organización de Comercio respecto al acuerdo general sobre tarifas y servicios de educación superior (Cortés Chávez, 2010).

Estos dos eventos supra mencionados remiten a la conceptualización de la educación superior como un servicio y no como un derecho que tiene implicancias en la función que se atribuye a las instituciones de nivel superior. En las antípodas de ese razonamiento se encuentra una perspectiva que promueve tener instituciones justas, equitativas e igualitarias con un fuerte compromiso social, que implica posicionarse en el derecho a la educación como un derecho humano, que actúa además como garante del complimiento de otros derechos y que no necesariamente tiene que ver con la prestación de un servicio y la rentabilidad que tal prestación implica.

Sin embargo, debe reconocerse que la internacionalización de la educación también tiene un aspecto imprescindible en la medida que ha realizado un gran aporte en un sentido colaborativo y constatativo que el avance del conocimiento científico requiere y está es quizás la principal razón que motiva a gobiernos e IES a suscribir convenios y acuerdos de colaboración reciproca en el que las partes intervinientes se vean mutuamente favorecidas.

En la evaluación de la calidad de las instituciones de educación superior, igualmente, cumple un rol fundamental la internacionalización de las instituciones. De hecho, la mayoría de los rankings de universidades toman en cuenta este indicador de alumnos y docentes e investigadores extranjeros. Sin embargo, debe reconocerse que también, merced a los avances tecnológicos en la comunicación proliferaron las ofertas de servicios educativos cuya calidad ni siquiera puede mencionarse porque no se puede constatar los mecanismos de evaluación y acreditación que tienen esos programas que terminan expidiendo títulos. La calidad esa formación debiera es cuanto menos cuestionable dado que no se conocen las agencias gubernamentales o privadas que terminan interviniendo en la acreditación de esos estudios y en la fiabilidad que esos procesos de evaluación; para los casos en los que son sometidos. Existen propuestas de estudios en los que ni siquiera puede constatarse que sean sometidos a algún proceso de evaluación y acreditación ni en los países de origen ni en los de destino. Esto obliga a que los países actualicen su marco normativo respecto a la validez de esos títulos y a la regulación de esas actividades en el ámbito local.
Esta situación adquiere tal dimensión que incluso se esgrime el concepto de "Internationalization at Home" (IaH) o Internacionalización en Casa (IeC), que hace referencia a cualquier actividad internacional o intercultural que desarrolle una institución de educación superior, a excepción de los intercambios salientes (BELÉN. 2011).

La movilidad estudiantil es solo un aspecto de la movilidad académica que implica la movilidad de profesores e investigadores. Las instituciones, los gobiernos y organismos descentralizados, fundaciones e instituciones de diversos países promueven este tipo de movilidad, viéndose intensificada esta actividad en los últimos años con la proliferación de propuestas que promovían el intercambio y la movilidad académica que solo para mencionar algunas se pueden citar a las convocatorias de Columbus, Erasmus, Fulbright, CAPES. SPU, OEI, AUIP, AECI, NEIES - MERCOSUR, encargadas por gobiernos, por las propias instituciones de educación superior o por organismos supra nacionales tales como UNESCO, OCDE, Banco Mundial, PNUD, OEA o el BID.

\section{METODOLOGÍA}

En este trabajo se utilizan datos provenientes del Centro de Datos, del Instituto de Estadística de la UNESCO (UIS). Dicho instituto posee una plataforma que permite la extracción de datos e indicadores ya elaborados por los países. Al tratarse de una base de datos secundarios, la reconstrucción de los indicadores por cuenta propia se ve dificultada por no contar con la información de primera fuente que sirvió de base para la construcción de los mismos. No obstante; estos indicadores poseen la ventaja de estar estandarizados, evento que posibilita la comparación internacional. La fuente seleccionada tiene como principal ventaja la comparabilidad de los datos que para la metodología comparativa adoptada resulta optima.

Se consideraron para el estudio comparativo principalmente el número de estudiantes movilizados hacia o desde el país de origen. Además del número de estudiantes que los países remiten o reciben se tomó en consideración dos indicadores: La tasa bruta de entrantes o ingresos al país, entendida el número de estudiantes extranjeros como porcentaje de la matrícula terciaria total en el país anfitrión. Al respecto deberá señalarse que al tomar en cuenta la matricula del país de destino nos da una idea de lo que los estudiantes extranjeros representan en relación al total de estudiantes.

Otro indicador seleccionado fue Tasa bruta de matrícula de salida que refiere al número total de estudiantes de un país determinado que estudian en el extranjero, 
expresado como porcentaje de la población en edad terciaria en ese país. Este indicador nos da una idea de la cantidad de alumnos que estudian afuera de la población que está en edad de realizar teóricamente estudios terciarios.

Este artículo está focalizado sobre seis países de la región, Argentina, Brasil, Colombia, Chile, Paraguay y Uruguay. La elección de los mismos se realizó, fundamentalmente, por poseer los mismos indicadores para los años en los que se propuso la comparación. Además, estimamos que al poseer estos países contextos sociales y económicos distintos, con tradiciones y culturas diferentes, la comparación de los indicadores puede resultar relevante para la diagramación de políticas educativas apropiadas a la realidad de cada país.

Lamentablemente la serie de datos se encuentra incompleta para los países analizados y tanto Paraguay como Uruguay no disponen de datos de referidos a los alumnos residentes en ese país de otra nacionalidad. Además, la fuente no permite precisar algunas otras cuestiones por ejemplo para el caso de individuos con doble nacionalidad, evento que posiblemente disminuya el registro del dato.

\section{RESULTADOS}

Los resultados se presentan por país y en los gráficos se ilustran solo los diez principales países de origen o destino. Las escalas de los gráficos son diferentes debido la variabilidad de los datos.

De los indicadores aquí seleccionado, somo se explica en el apartado metodológico solo se mencionan dos tasas para dar cuenta del flujo de ingreso y de egreso de la población estudiantil.

El primer país en ser analizado, siguiendo solo un criterio de orden alfabético es Argentina. Este país es em términos generales un país receptor de estudiantes, mayoritariamente de países limítrofes. La información extraída em el año 2019 muestra que el país era anfitrión de 75688 estudiantes, mientras que matriculaba 8371 alumnos fuera del país, aproximadamente un $10 \%$ del total de los estudiantes movilizados hacia o desde este país, convirtiéndose con ello em un país que revierte un poco la polaridad sur norte que tienen los países de América latina hacia los grandes polos de saber en Norte América o en Europa primordialmente. Sin lugar a dudas el acceso a las universidades públicas y gratuitas cumplen un rol fundamental y principal atractivo de los estudiantes limítrofes. Los gráficos solo ilustran a los diez principales países.

Un indicador interesante de mencionar es la tasa de movilidad entrante, entendida como el número de estudiantes extranjeros que estudian en un país determi-
Figura 1: Movilidad de Estudiantes hacia y desde Argentina.

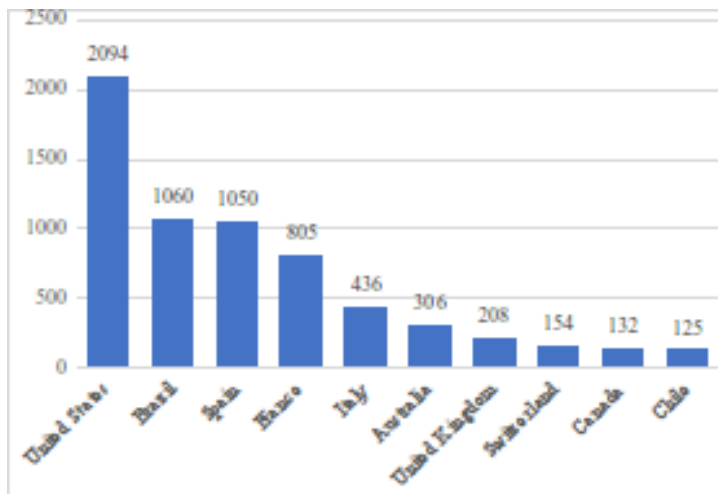

(a)

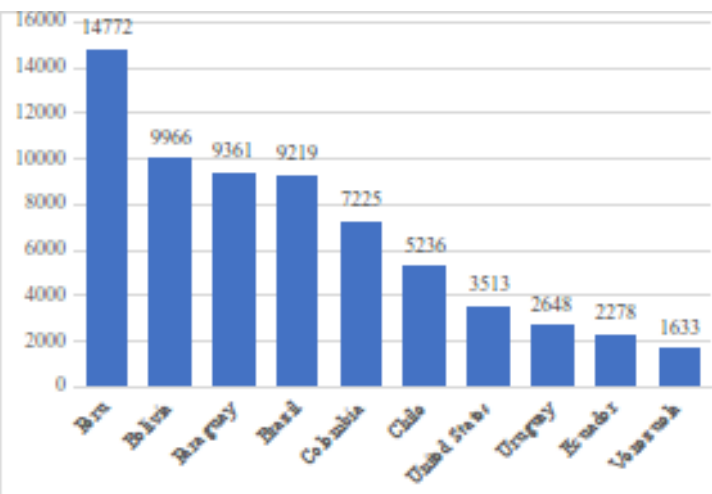

(b)

nado, expresado como porcentaje de la matrícula terciaria total en ese país. En argentina ese valor asciende a 2.5.

Otro indicador importante de mencionar es la tasa bruta de matrícula de salida, entendida como el número total de estudiantes de un país determinado que estudian en el extranjero, expresado como porcentaje de la población en edad terciaria en ese país. El indicador en Argentina es de 0.2.

Brasil, por su parte, muestra un total de 52515 estudiantes movilizados hacia algún país extranjero, mientras que aloja a 19380 estudiantes extranjeros. La gran cantidad de alumnos movilizados de la principal economía de la región es importante por cuanto inmediatamente nos remite al interrogante de la formación de la elite intelectual y económica y los intereses que pueden estar comprometidos. Un dato relevante del país en cuestión es que tiene como principales destinatarios de sus instituciones de nivel superior a estudiantes originarios de Angola, y luego de países limítrofes encabezados por estudiantes colombianos, seguidos de peruanos, 
INTERNACIONALIZACIÓN DE LAS INSTITUCIONES DE NIVEL SUPERIOR. FLUJOS DE MOVILIDAD ESTUDIANTIL.

Tabela 1: Indicadores seleccionados de movilidad estudiantil por país.

\begin{tabular}{|c|c|c|c|c|c|c|}
\hline Indicador & Argentina & Brasil & Paraguay & Colombia & Chile & Uruguay \\
\hline \multicolumn{7}{|c|}{ Estudiantes en el extranjero: } \\
\hline Número total de estudiantes emigrantes & 8371 & 52515 & 12435 & 36626 & 14122 & 4630 \\
\hline Porcentaje del total de estudiantes migrantes & 0,2 & 1 & 0,2 & 0,7 & 0,3 & 0,1 \\
\hline Tasa de movilidad hacia el extranjero & 0,3 & 0,6 & & 1,5 & 1,1 & 3,1 \\
\hline Tasa bruta de matrícula en el extranjero & 0,2 & 0,3 & 1,8 & 0,9 & 1 & 1,7 \\
\hline \multicolumn{7}{|c|}{ Estudiantes Alojados: } \\
\hline Número total de estudiantes extranjeros alojados & 75688 & 19996 & & 4550 & 4708 & \\
\hline porcentaje del total de estudiantes migrantes & 1,5 & 0,4 & & 0,1 & 0,1 & \\
\hline Tasa de movilidad hacia el país anfitrión & 2,5 & 0,2 & & 0,2 & 0,4 & \\
\hline
\end{tabular}

Fuente: Elaboración propia en base a datos extraído de UIS UNESCO.

Figura 2: Movilidad de Estudiantes hacia y desde Brasil.

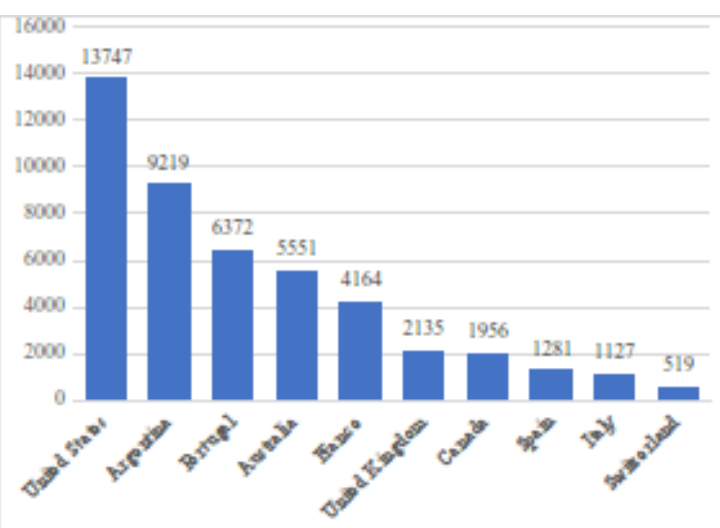

(a)

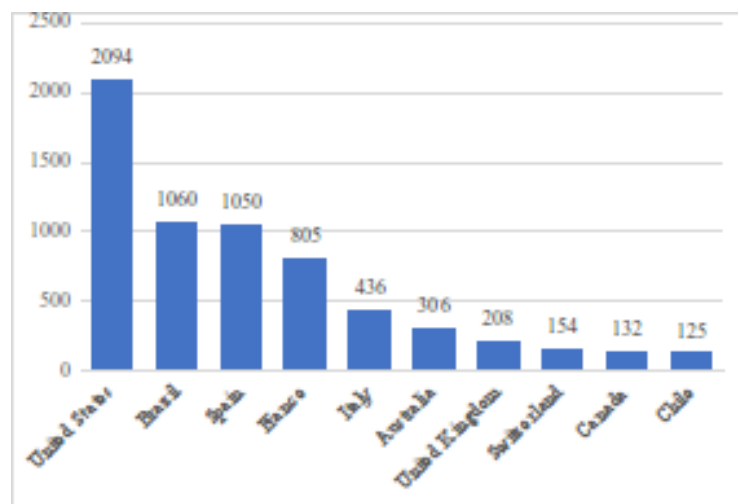

(b)

paraguayos y argentinos.

La tasa de movilidad entrante en Brasil es de 0.2.

El sistema de ingreso, desempeña nuevamente un papel fundamental. La tasa bruta de matrícula de salida en Brasil es de 0.3.

Lamentablemente no se registran datos del número de alumnos que el sistema educativo de Paraguay regis-
Figura 3: Movilidad de Estudiantes desde Paraguay.

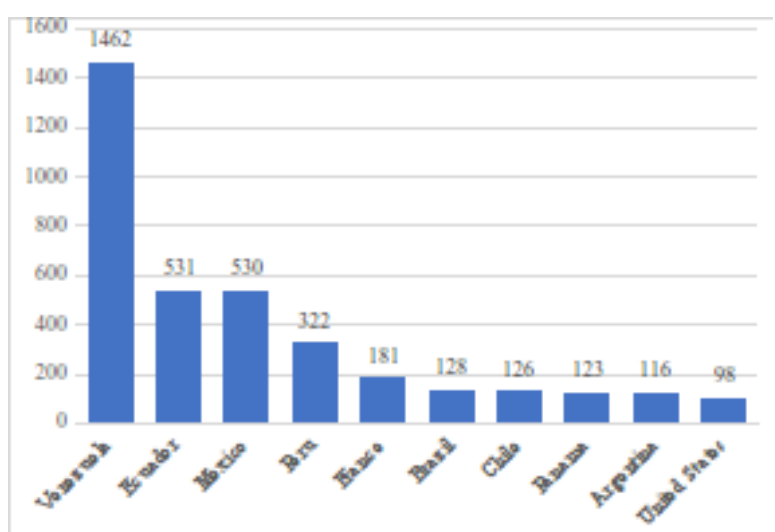

tra como provenientes de otras regiones.

Respecto al destino de los estudiantes de Paraguay, los Estados Unidos de Norteamérica resulta el principal destino, seguidos por Argentina como lugar elegido y sucedido por otros lugares de Europa, aunque destaca Australia como destino.

La tasa de movilidad entrante lógicamente al carecer de datos no se puede estimar, pero si debe reconocerse al país como expulsor de población. La tasa bruta de matricula en el extranjero es la más alta de los países de la región alcanzando la cifra de 1.8 .

Colombia también muestra un flujo en el que envían más estudiantes en el extranjero de los que recibe. Los gráficos tienen diferentes escalas con fines ilustrativos y muestra solo los datos de los diez principales países, pero debe señalarse que el país registra 36626 estudiantes connacionales en algún país extranjero. Entre los estudiantes que reciben sobresalen los provenientes de Venezuela, quienes por proximidad geográfica y por cuestiones de índole social, políticas y económicas se han convertido en un tópico que requiere de un análisis que excede el aquí propuesto. En total se registran como alumnos extranjeros en el país 4550 , apenas un $11 \%$ del 


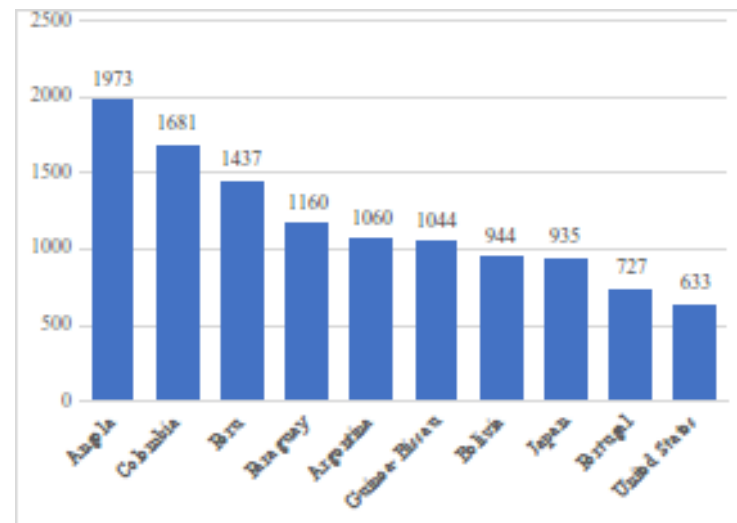

(a)

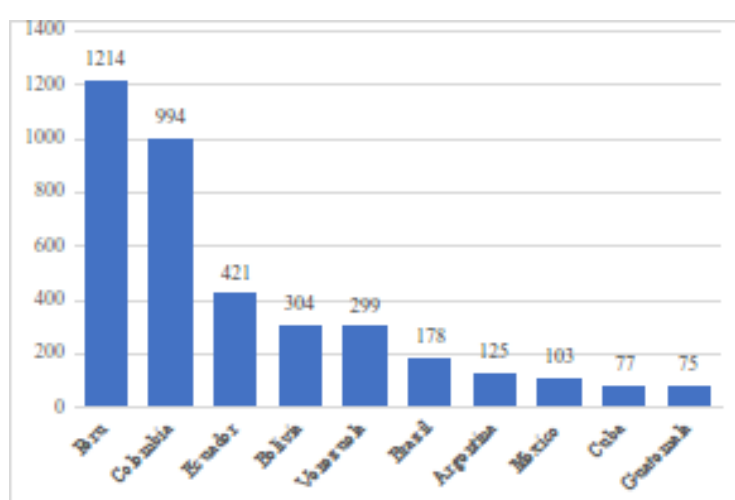

(b)

total de alumnos movilizados. La tasa bruta de salida es de 0.9 .

Chile remite a países extranjeros a realizar estudios superiores a 14122 individuos y recibe a 4708 alumno. Se trata, nuevamente, como la mayoría de los países de la región a excepción de Argentina de una situación en la que claramente se envían más estudiantes de los que reciben. En este caso reciben estudiantes de Perú, en primer termino y luego de otros países de la región destacándose los estudiantes colombianos. En lo atinente al destino de elección de los estudiantes chilenos, Argentina, resulta su primera opción, encontrándose matriculados 5236 alumnos de esa nacionalidad, lo que representa solo en ese país más que la cantidad de alumnos que el país trasandino recibe de la totalidad de los otros países. El resto de los países de destino de los estudiantes chilenos es principalmente EEUU seguido de algunos países europeos y Australia nuevamente, que pesé a la distancia de la región, al igual que Canadá aparece como destino predilecto para los estudiantes la-

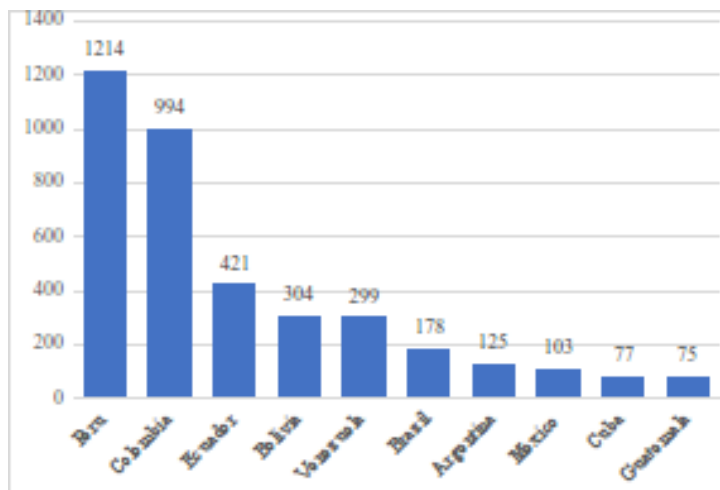

(a)

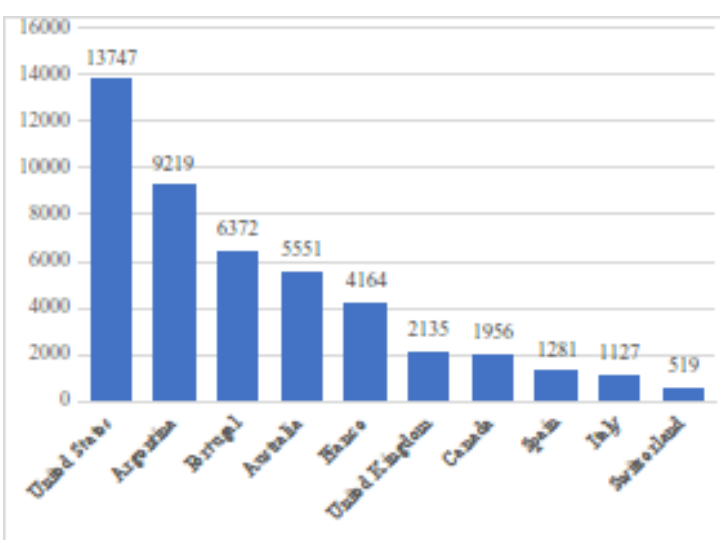

(b)

tinos.

La tasa de movilidad de entrantes en este país es 0,4 , mientras que la tasa bruta de matricula en el extranjero de 1 .

Figura 6: Movilidad de Estudiantes desde Uruguay.

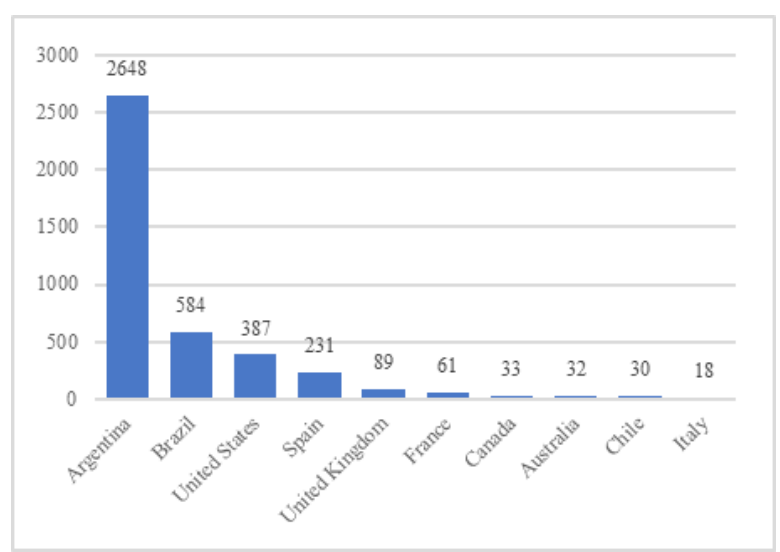


Al igual que para el caso de Paraguay, Uruguay no tiene datos de alumno extranjero en su país, al menos desde la fuente de datos que se está consultando. De lo que sí se tiene noción es del número de estudiantes que se encuentran matriculados en otros países y para cuyo caso la fuente consultada dice que se tratan de 4630 individuos. El 57\% de esos alumnos están radicados en su vecino país de Argentina, con quien comparte además una identidad cultural muy próxima. El resto de los países elegidos por los uruguayos es similar a lo observado para el resto de los países de América latina.

La tasa bruta de matricula en el extranjero es una de las más altas de la región, sólo superada, en los países bajo estudio por Paraguay, por apenas una décima con una tasa de 1.7.

\section{CONCLUSIONES}

La internacionalización de las IES es un fenómeno presente en los países de la región y la movilidad académica se encuentra presente y convive con otras propuestas transnacionales por la cual es inminente una regulación y tratado por parte de los países de Latinoamérica para regular su funcionamiento.

Un elemento fundamental a considerar en los países respecto a los flujos migratorios es el sistema de ingreso a las instituciones de estudios superiores, además de las erogaciones económicas que demandan tales países para realizar algunas de sus propuestas o programas de carreras de grado y posgrados. En países cuyo sistema de ingreso es más flexible como es el caso de Argentina, lógicamente, el ingreso a los estudios superiores se produce de manera masiva para locales y foráneos. Está es quizás una de las razones principales por las cuales Argentina es un polo de atracción para los países de la región contrario a lo que acontece con el resto de los países de la región que envían muchos más estudiantes de los que reciben. Sobre este hecho en particular destaca el volumen de estudiantes brasileros, que si bien refieren a una población de más de 200 millones de habitantes no deja de ser impresionante porque implica que la economía más importante de Latinoamérica envía a un número importante de sus jóvenes a realizar sus estudios a otros países.

El resto de los países de la región envían principalmente sus estudiantes a los países europeos a los que se encuentra vinculado culturalmente o hacia los Estados Unidos de América por el prestigio de sus universidades, destacándose que entre los principales destinos aparecen además Canadá y Australia como países receptores de la población estudiantil. Para el caso de Brasil destaca que recibe entre sus principales estudiantes a personas provenientes del áfrica, principalmente de Angola y Guinea-Bisáu, ambos países ex colonias portuguesas, situación que no se observa en esa magnitud en el resto de los países.

El flujo de migración general que tienen los países no dista mucho de lo que se observa en la población estudiantil, en todo caso ilustra que las razones que movilizan a una persona en búsqueda de una mejor educación, sea la migración temporaria o permanente es un fuerte motivo de emigración.

\section{REFERÊNCIAS}

AUPETIT, S. D. La internacionalización de la educación superior en américa latina: oportunidades y desafíos. In: Conferencia dictada en el Pabellón Argentina de la Ciudad Universitaria, Córdoba. Cordoba: [s.n.], 2007. v. 21.

BELÉN, J. La internacionalización en casa en una perspectiva global: un estudio crítico del informe del 3. er estudio global de la aiu. Revista de Universidad y Sociedad del Conocimiento, v. 1, n. 2, p. 85-100, 2011.

\section{C., G. C. La internacionalización de la Universidad. \\ 1. ed. Bogotá Colombia: Umbral Científico, 2003.}

Cortés Chávez, S. Tendencias de la internacionalización en las IES públicas de América Latina. 1. ed. Ajusco: Universidad Pedagógica Nacional, 2010.

GÉRARD, E.; MALDONADO, E. "polos de saber"y"cadenas de saber": Impactos de la movilidad estudiantil en la estructuración del campo científico mexicano. Revista de la educación superior, Asociación Nacional de Universidades e Instituciones de Educación Superior, v. 38, n. 152, p. 49-62, 2009.

HERMO, J. P.; PITTELLI, C. La creciente importancia de la movilidad estudiantil transnacional: América látina y la ocde en visión comparada. In: UFSC. IX Colóquio Internacional sobre Gestão Universitária na América do Sul. Florianópolis, 2009. p. 1-14.

KURI, R. G. La internacionalización de las universidades en méxico. Revista de la educación superior, Asociación Nacional de Universidades e Instituciones de Educación Superior, v. 35, n. 137, p. 133-142, 2006.

\section{LUCHILO, L. La medición de la movilidad internacional de estudiantes de educación superior. 2006.}

PRADOS, M. L. La internacionalización de la universidad: debates globales, acciones regionales. 
Revista Brasileira de Educação, SciELO Brasil, v. 23 , n. 1, p. 1, 2018.

SEBASTIÁN, J. La internacionalización de las universidades como estrategia para el desarrollo institucional. Innovación educativa, Instituto Politécnico Nacional, v. 5, n. 26, p. 1-11, 2005.

SEBASTIÁN, J. Dimensiones y métrica de la internacionalización de las universidades. Universidades, Unión de Universidades de América Latina y el Caribe, v. 1, n. 51, p. 3-16, 2011.

UNESCO. ISCED 2011 Operational Manual Guidelines for classifying national education programmes and related qualifications. 2011.

UNESCO. International Standard Classification of Education ISCED 2011. 2012. Montreal Quebec.

UNESCO. ISCED Fields of Education and Training 2013 (ISCED-F 2013). 2013.

UNESCO. International Standard Classification of education. Fields of education and training 2013

(ISCED-F 2013) - Detailed field descriptions. 2015.

UNESCO. UIS Indicator Development in the Field of Education. 2017. 